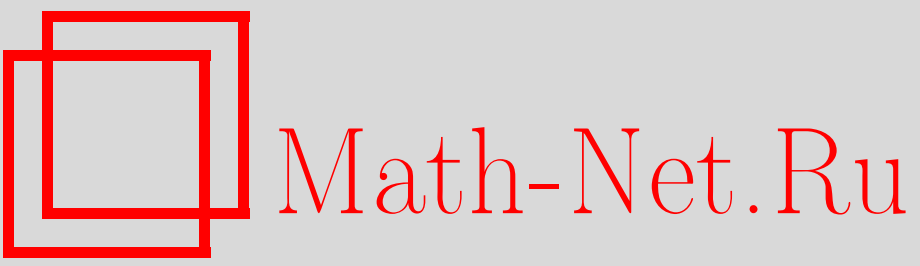

М. И. Штогрин, Неизгибаемость квадрильяжа кренделя, УМН, 1999, том 54, выпуск 5, 183-184

DOI: https://doi.org/10.4213/rm218

Использование Общероссийского математического портала Math-Net.Ru подразумевает, что вы прочитали и согласны с пользовательским соглашением

http://www.mathnet.ru/rus/agreement

Параметры загрузки:

IP: 3.89 .197 .203

26 апреля 2023 г., 14:00:14 


\title{
НЕИЗГИБАЕМОСТЬ КВАДРИЛЬЯЖА КРЕНДЕЛЯ
}

\author{
М. И. Штогрин
}

Основные понятия и определения, использованные ниже, даны в работах [1], [2], в которых была доказана решеточность и неизгибаемость квадрильяжа $\mathbb{Q}^{2}$, гомеоморфного сфере или тору, квадрильяжно отображенного в $\mathbb{R}^{3}$ с погружением реберного остова квадрильяжа $\mathbb{Q}^{2}$.

ТЕОрема. Пусть $\mathbb{Q}^{2}$ - произвольный квадрильяж кренделя. Тогда любое квадрильяжное отображение $f: \mathbb{Q}^{2} \rightarrow \mathbb{R}^{3}$, при котором реберный остов $\mathbb{Q}^{2}$ погружен в $\mathbb{R}^{3}$, решеточно.

СлЕдСтвиЕ. В условиях теоремы образ квадрильяжа кренделя $\mathbb{Q}^{2}$ в $\mathbb{R}^{3}$ неизгибаем.

В работе [2] определен граф стыљовки, разбивающий поверхность квадрильяжа $\mathbb{Q}^{2}$ на связные компоненты, называемые гранями. Каждая грань принадлежит своей кубической решетке и имеет не менее 4 сторон. В каждой вершине сходятся не менее 4 граней. Для разбиения поверхности на эти грани по формуле Эйлера имеем неравенство $4 \chi \leqslant-N$, где

$$
N=\sum(q-4) \beta_{q}+\sum(n-4) 2_{n} .
$$

Так как на продолжении каждого ребра графа стыковки имеется вершина валентности не менее 5 , а все выходящие из нее ребра графа стыковки разно направлены (в силу погружения реберного остова $\mathbb{Q}^{2}$ в $\mathbb{R}^{3}$ ), то $N \geqslant(5-4) 6=6$. Для сферы или тора этой оценки более чем достаточно, см. [2]. Для кренделя ниже будет дана более сильная оценка $N \geqslant 9$.

Лемма. Пусть в точке $О$ сходятся пять разно направленных отрезков, записанных в чиклической последовательности. Пусть плоский угол между любыми двумя соседними отрезками последавательности равен $\pi / 2$. И пусть двугранный угол межсду любыми двумя смежными по общей стороне прямоугольниками, построенными на соседних отрезках, не равен $\pi / 2$. Тогда вершина любого прямоугольника, противоположная вершине $O$, не совпадает с концом любого из данных пяти отрезков.

Введем обозначения: $(O A, O B, O C, O D, O E)$ - циклическая последовательность отрезков, удовлетворяющих условиям леммы, $M$ - вершина построенного на отрезках $O A$ и $O B$ прямоугольника, противоположная вершине $O$. Так как отрезки разно направлены, то их концы лежат в разных точках пространства. Точка $M$ не совпадает с $O, A, B$, как четвертая вершина прямоугольника $O A M B$. Ребро $O C$ прямоугольника, смежного с $O A M B$ по стороне $O B$, перпендикулярно $O B$, и поэтому $M$ не совпадает с $C$. Аналогично, $M$ не совпадает с $E$. Предположим, что $M$ совпадает с $D$. Тогда $O M$ совпадает с $O D$ и ввиду $O C \perp O D$ получим $O C \perp O M$. Но $O C \perp O B$, поэтому $O C$ перпендикулярно плоскости треугольника $\triangle O B M$, в которой лежит $O A$. Поэтому $O C \perp O A$. Двугранный угол между прямоугольниками, смежными по стороне $O B$, измеряется плоским углом $\widehat{A O C}$, равным $\pi / 2$. Это противоречит условию леммы. Следовательно, $M$ не может совпадать с $D$. Лемма доказана.

ЗАмечание 1 . Вершины $M_{1}, M_{2}, M_{3}, M_{4}, M_{5}$ фигурирующих в лемме прямоугольников, противоположные $O$, расположены по меньшей мере в 3 различных точках пространства.

В самом деле, вершшны $M_{i}$ и $M_{j}$ смежных по стороне двух прямоугольников не совпадают, так как иначе две их стороны, перпендикулярные общей стороне, оказались бы одинаково направленными, что противоречило бы условиям леммы. Так как среди любых 3 из данных 5 прямоугольников всегда найдутся 2 прямоугольника, смежные по общей стороне, то никакие 3 из 5 точек $M_{k}$ не могут находиться в одной и той же точке пространства. Ввиду этого в одной точке пространства может находиться не более 2 из 5 точек $M_{k}$.

ЗАмечАниЕ 2. Справедливость леммы не нарушится, если в формулировке леммы вместо пяти разно направленных отрезков взять шесть.

Работа выполнена при финансовой поддержке Российского фонда фундаменталњных исследований (грант № 99-01-00010). 
ПРЕДЛОЖЕнИЕ. Пусть $\mathbb{Q}^{2}$ - квадрильяж двумерной поверхности произвольного рода. Пусть при квадрильяжном отображении $f: \mathbb{Q}^{2} \rightarrow \mathbb{R}^{3}$ реберный остов $\mathbb{Q}^{2}$ погружен в $\mathbb{R}^{3}$. И пусть граф стыковки не пуст. Тогда $N \geqslant 9$.

1. Пусть $\theta_{q} \geqslant 1$ при некотором $q \geqslant 7$. Тогда на продолжении каждого ребра в связке из $q$ ребер есть вершина валентности (степени) не менее 5 . Поэтому $N \geqslant(5-4) 7+(7-4) 1=10$.

2. Пусть $\theta_{q}=0$ при всех $q \geqslant 7, \theta_{6} \geqslant 2$. Тогда $\theta_{5} \neq 0$ и $N \geqslant(5-4) 5+(6-4) 2=9$.

3. Пусть $\theta_{q}=0$ при всех $q \geqslant 7, \theta_{6}=1,2_{n} \geqslant 1$ при некотором $n \geqslant 5$. Тогда $\theta_{5} \neq 0$ и $N \geqslant(5-4) 6+(6-4) 1+(5-4) 1=9$.

4. Пусть $\theta_{q}=0$ при всех $q \geqslant 7, \theta_{6}=1,2_{n}=0$ при всех $n \geqslant 5$. Тогда каждая грань ограничена прямоугольником и $\theta_{4}=0$ (иначе у двух граней угол при 4-валентной вершине равнялся бы $\pi$, у них число сторон превысило бы 4, что противоречило бы предположению). Следовательно, все вершины лишь 6- и 5-валентные. Связка из 6 ребер графа стыковки удовлетворяет лемме, с учетом замечания 2 . Поэтому $N \geqslant(5-4) 7+(6-4) 1=9$.

5. Пусть $\beta_{q}=0$ при всех $q \geqslant 6$, 乙n $_{n} \geqslant 1$ при некотором $n \geqslant 7$. Тогда $\theta_{5} \neq 0$ и $N \geqslant(5-4) 6+$ $(7-4) 1=9$.

6. Пусть $\theta_{q}=0$ при всех $q \geqslant 6, \imath_{n}=0$ при всех $n \geqslant 7, \imath_{6} \geqslant 2$. Тогда $\theta_{5} \neq 0$ и $N \geqslant(5-4) 6+$ $(6-4) 2=10$.

7. Пусть $\theta_{q}=0$ при всех $q \geqslant 6, \imath_{n}=0$ при всех $n \geqslant 7,2_{6}=1$ и $2_{5} \geqslant 1$. Тогда $8_{5} \neq 0$ и $N \geqslant(5-4) 6+(5-4) 1+(6-4) 1=9$.

8. Пусть $\theta_{q}=0$ при всех $q \geqslant 6, \imath_{n}=0$ при всех $n \geqslant 7,2_{6}=1$ и $2_{5}=0$. Тогда $8_{4}=0$ (иначе у двух граней угол при 4-валентной вершине был бы равен $\pi$, две грани имели бы более четырех сторон, что противоречило бы предположению). Следовательно, все вершины 5 -валентные. Возьмем вершину 6-сторонней грани (она лежит в кубической решетке), в которой ее стороны составляют угол $\pi / 2$. Связка из пяти ребер с этой вершиной удовлетворяет лемме. В качестве вершины $M$ возьмем вершину одного из четырех прямоугольников, которые лежат на краю своих граней. Получим $N \geqslant(5-4) 7+(6-4) 1=9$.

9. Пусть $\theta_{q}=0$ при всех $q \geqslant 6$, 2n $_{2}=0$ при всех $n \geqslant 6, \beta_{4} \geqslant 2$. Тогда $25 \geqslant 4$ (с каждой 4 -валентной вершиной инцидентны по два 5-сторонника), в5 $\neq 0$. Поэтому $N \geqslant(5-4) 6+$ $(5-4) 4=10$.

10. Пусть $\theta_{q}=0$ при всех $q \geqslant 6,2_{n}=0$ при всех $n \geqslant 6, \theta_{4}=1$. Тогда $25=2$. Кроме $8_{4}=1$ все остальные вершины 5-валентные. В 4 -валентной вершине сходятся два 4-сторонника и два 5-сторонника. Циклическая последовательность радиусов звезды $\mathrm{St} O$, направленных вдоль ребер граффа стыковки в 4-валентной вершине $O$, имеет вид $(\mathbf{i}, \mathbf{j},-\mathbf{j},-\mathbf{i})$. Эти радиусы разбивают звезду St $O$ на 4 сектора $(O, \mathbf{i}, \mathbf{j}),(O, \mathbf{j},-\mathbf{j}),(O,-\mathbf{j},-\mathbf{i}),(O,-\mathbf{i}, \mathbf{i})$. Каждый сектор раположен в своей грани. Один 4-сторонник имеет 5 -валентные вершины $x \mathbf{i}, y \mathbf{j}, x \mathbf{i}+y \mathbf{j}$. Другой 4-сторонник имеет 5-валентные вершины $-u \mathbf{i},-v \mathbf{j},-u \mathbf{i}-v \mathbf{j}$. Один из 5-сторонников прибавляет две свои 5 -валентные вершины $x \mathbf{i}+w(\mathbf{j} \cos \gamma+\mathbf{k} \sin \gamma)$ и $-u \mathbf{i}+w(\mathbf{j} \cos \gamma+\mathbf{k} \sin \gamma)$. Здесь $x, y, u, v, w \in \mathbb{N}$. Поэтому $N \geqslant(5-4) 8+(5-4) 2=10$. Или же $N \geqslant 9$, как в п. 8 .

11. Пусть $\theta_{q}=0$ при всех $q \geqslant 6, \imath_{n}=0$ при всех $n \geqslant 6,8_{4}=0$. Тогда $85 \neq 0,2_{5}=0$ и и силу замечания 1 имеем $N \geqslant(5-4) 9=9$.

ДокАЗАТЕЛЬСтво ПРЕДЛОЖЕНИЯ. Так как непустой граф стыковки имеет вершину, в которой сходится не менее 5 его ребер (т.е. всегда существует $q \geqslant 5$ ), то для $q \geqslant 4$ и $n \geqslant 4$ в пп. 1-11 перечислены все мыслимые возможности. Поэтому для поверхности любого рода имеет место оценка $N \geqslant 9$. Предложение доказано.

ДокАЗАТЕЛЬСтво теОРемЫ. В силу предложения при $\chi=-2$ соотношение $4 \chi \leqslant-N$ невозможно. Следовательно, при $\chi=-2$ граф стыковки пуст. Теорема доказана.

\section{СПИСОК ЛИТЕРАТУРЫ}

[1] Долбилин Н. П., Штанько М. А., Штогрин М. И. // Докл. АН. 1997. Т. 354. № 4. С. 443-445. [2] Долбилин Н. П., Штанько М. А., Штогрин М. И. // УМН. 1999. Т. 54. №4. C. $167-168$. 